\title{
B-lymphocytic infiltration of the prostate to a patient with chronic lymphocytic leukemia. A case report
}

\author{
Ioannis Anastasiou, Spyridon Skoufias, Ioannis Katafigiotis, Ioannis Skarmoutsos, \\ Constantinos A. Constantinides \\ ${ }^{1} 1^{\text {st }}$ University Urology Clinic, Laiko Hospital, Athens, Greece.
}

\begin{abstract}
Summary Involvement of the prostate gland, as an early extra-nodal manifestation of a

hematologic disease, or as a secondary infiltration is rare. Even rarer is the acute urinary retention due to infiltration by lymphocytes and simultaneously enlarged prostate. We present a case of a 61 years old male patient with a history of chronic lymphocytic leukemia, who was under oncological follow-up with no active treatment and had typical lower urinary tract symptoms due to benign prostatic hyperplasia and was receiving 5-alpha reductase inhibitor. After an acute urinary retention episode which was managed with a suprapubic catheter due to urethral catheter insertion failure, the patient was submitted to a transurethral prostatectomy. Histological examination revealed lymphocytic infiltration of the prostatic parenchyma by mostly small B cells. B-lymphocytic infiltration of the prostate gland, causes symptoms similar to benign prostatic hyperplasia. Acute urinary retention due to B-lymphocytic infiltration of the prostate is rare and the diagnosis is always histological and an oncological re-evaluation is necessary.

The prognosis of these patients is related to the generalized disease rather than to the prostatic involvement.
\end{abstract}

KEY WORDS: B-lymphocytic infiltration; Prostate; Chronic lymphocytic leukemia.

Submitted 12 December 2016; Accepted 11 January 2017

\section{INTRODUCTION}

Hematological diseases such as lymphomas and chronic lymphocytic leukemia rarely include the prostate gland as an extranodal manifestation and even rarer is their first appearance to be in the lower urinary tract. We present a 61 years old patient with a history of chronic lymphocytic leukemia who underwent transurethral resection of prostate adenoma (TUR-P) due to an acute urinary retention.

Histological examination showed low B-lymphocytic infiltration of the prostate parenchyma of mainly small lymphocytes.

\section{Case report}

A 61 years old patient with a history of chronic lymphocytic leukemia, diagnosed seven years ago, without receiving any treatment underwent transurethral resection of prostate adenoma.

The patient had lower urinary tract symptoms during the last months due to benign prostate hyperplasia and was receiving treatment with dutasteride (5-a reductase inhibitor). His IPSS score was 21 and he also had an abnormal uroflow with a maximum flow of $8 \mathrm{ml} / \mathrm{sec}$. His serum prostate-specific antigen was $1.4 \mathrm{ng} / \mathrm{ml}$.

Ultrasonography showed an intravesical prostate protrusion. After six months of treatment with dutasteride the patient suffered from an acute urinary retention, which was treated with suprapubic catheter placement, due to transurethral catheterization failure. Preoperatively his blood count was pathological with $37.000 / \mathrm{mm}^{3}$ WBC. He underwent transurethral prostate resection and the postoperative course was uneventful. He was dismissed after a three-day hospitalization with $35 \mathrm{ml}$ of post voiding residual. Histological examination showed that some of the prostate chips were infiltrated by lymphocyte cells (Figure 1).

That cell population was composed by mainly small cells without proliferation centers. The presence of a few larger cell types was also noticed. Immunohistochemistry showed that this lymphocyte population was positive in staining for CD20, CD5, CD23 and negative for cyclinD1 and CD3 (Figure 1).

B-lymphocytic infiltration of the prostate was consistent with patients' history of chronic lymphocytic leukemia. Patient was then referred for oncology reevaluation and followed a therapeutic protocol with filgrastim, EPO and rituximab.
Figure 1.

Positive staining for CD5, CD20 and CD23 respectively.

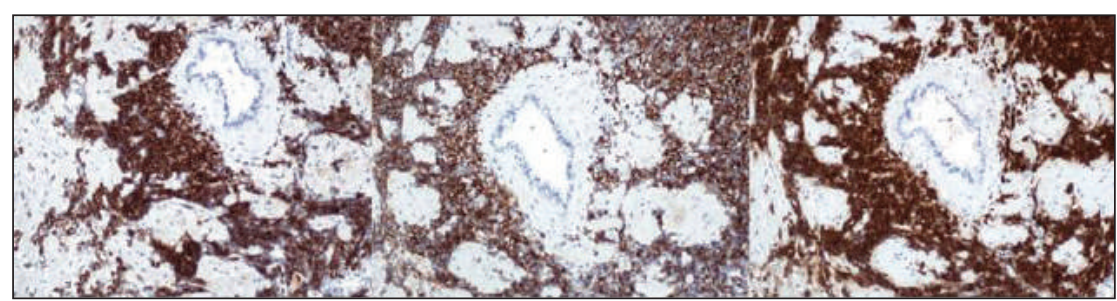




\section{Discussion}

Extra-nodal localization of hematological diseases, such as chronic lymphocytic leukemia and lymphomas rarely regards the genitourinary tract and especially prostate gland (1). In the majority of cases leukemic infiltration of the prostate is asymptomatic and serum prostate-specific antigen level is usually normal. Acute urinary retention is also rare, whilst in most of the cases prostate gland is not enlarged, and digital rectal examination mostly reveals a rubbery, firm, gland (2). Transabdominal and transrectal ultrasound examination may reveal some hypoechoic zones but these are not specific signs. Clinical diagnosis is difficult to made before surgery in patients without any history of malignancy. However in patients with a known history of hematological disease, LUTS might be indicative of prostate infiltration despite the fact, that prostate gland is not a common non-hematopoietic site of neoplastic cells spreading (3). TUR-P is usually performed to diagnose and release clinical symptoms due to lower urinary tract obstruction (1).

Chronic lymphocytic leukemia, as a disease of the elderly often coexists with BPH and symptoms usually don't subside with medical therapy such as $\alpha$-blockers and 5$\alpha$ reductase inhibitors.

Final diagnosis is set by histopathology examination and immunohistochemistry. The prognosis of these patients is related to the generalized disease rather than to the prostatic involvement. Oncological refer is necessary and most of the times, patients follow therapy protocols after the confirmation of leukemia secondary involvement.

\section{Conclusions}

Although it is rare, genitourinary tract can be an extranodal site of chronic lymphocytic leukemia. Therefore the differential diagnosis should always include the infiltration of the prostate gland by lymphocyte cells. Awareness is needed in the elderly men with hematological disease despite the fact that there are no specific lower urinary tract symptoms to indicate prostate infiltration. The diagnosis is always histological and an oncological reevaluation is necessary.

\section{REFERENCES}

1. Chu PG, Huang Q, Weiss LM. Incidental and concurrent malignant lymphomas discovered at the time of prostatectomy and prostate biopsy: a study of 29 cases. Am J Surg Pathol. 2005; 29:693-699.

2. Kassar O, Gouiaa N, Mdhaffar M, et al. Primary manifestation of small Lymphocytic Lymphoma in the Prostate: A case report. Tunis Med. 2015; 93:249-58.

3. D’Arena G, Guariglia R, Villani $O$, et al. An urologic face of chronic lymphocytic leukemia: sequential prostatic and penis localization Mediterr J Hematol Infect Dis. 2013; 5:e2013008.

\section{Correspondence}

Ioannis Anastasiou, MD

ekati2@otenet.gr

Spyridon Skoufias, MD

spyskouf@hotmail.com

Ioannis Katafigiotis, MD, MLS, PhD, FEBU (Corresponding Author)

katafigiotis.giannis@gmail.com

Ioannis Skarmoutsos, MD

iskarm@gmail.com

Constantinos A. Constantinides, MD

ckonstan@med.uoa.gr

$1^{\text {st }}$ University Urology Clinic, Laiko Hospital

Ag.Thoma 17, Athens 11527 - Greece 